\title{
Vortex lattices in Bose-Einstein condensates: from the Thomas-Fermi to the lowest Landau level regime
}

\author{
M. Cozzini, S. Stringari, and C. Tozzo \\ Dipartimento di Fisica, Università di Trento and BEC-INFM, I-38050 Povo, Italy
}

(Dated: March 13, 2021)

\begin{abstract}
We consider a periodic vortex lattice in a rotating Bose-Einstein condensed gas, where the centrifugal potential is exactly compensated by the external harmonic trap. By introducing a gauge transformation which makes the Hamiltonian periodic, we solve numerically the 2D Gross-Pitaevskii equation finding the exact mean field ground state. In particular, we explore the crossover between the Thomas-Fermi regime, holding for large values of the coupling constant, and the lowest Landau level limit, corresponding to the weakly interacting case. Explicit results are given for the equation of state, the vortex core size, as well as the elastic shear modulus, which is crucial for the calculation of the Tkachenko frequencies.
\end{abstract}

PACS numbers: 03.75.Kk, 03.75.Lm, 67.40.Vs

In the last years, a significant effort has been devoted to the study of vortex lattices in harmonically trapped rotating Bose-Einstein condensates. Striking results have been obtained experimentally, leading to the observation of large vortex lattices in fast rotating condensates $[1]$ and to the measurement of their dynamical properties 2, 3]. Theoretically, several predictions have been provided 4 , 5, 6] in agreement with experiments and more challenging regimes, related to the quantum Hall effect, have also been proposed [7].

In this paper we study the rotating analogue of a uniform Bose-Einstein condensate, where a periodic vortex lattice is present. For dilute atomic gases, which are highly compressible, this can only be obtained when the centrifugal potential is exactly cancelled by the harmonic confinement, i.e., when the harmonic trapping frequency is precisely equal to the angular velocity $\Omega$. Under this condition, in spite of the non-periodicity of the GrossPitaevskii (GP) equation governing the system, the density $n$ (see Fig. 囵) and the velocity field $\boldsymbol{v}^{\prime}$ in the rotating frame are periodic in the plane of rotation [8, 9], as can be proved by a proper gauge transformation. Analytical predictions are available in the literature for both the weakly interacting regime $6,10,11,12,13$, where the system is in the lowest Landau level (LLL), and the strongly interacting one [9], where the density is basically constant and the Thomas-Fermi (TF) approximation applies. Solving numerically the GP equation, we recover these limiting cases and compute the ground state for any value of the interaction strength. The validity of the mean-field approach requires that the number of atoms per vortex be very large. If this condition is not satisfied the system enters a strongly correlated regime related to the quantum Hall effect [7].

In the rotating frame, the external confinement $V_{\text {ext }}$ combines with the centrifugal potential, giving rise to the effective trapping $V_{\text {eff }}=V_{\text {ext }}-m \Omega^{2}\left(x^{2}+y^{2}\right) / 2$, where $m$ is the atomic mass. As anticipated above, we impose the compensation $V_{\text {eff }}=0$ and decouple the motion in

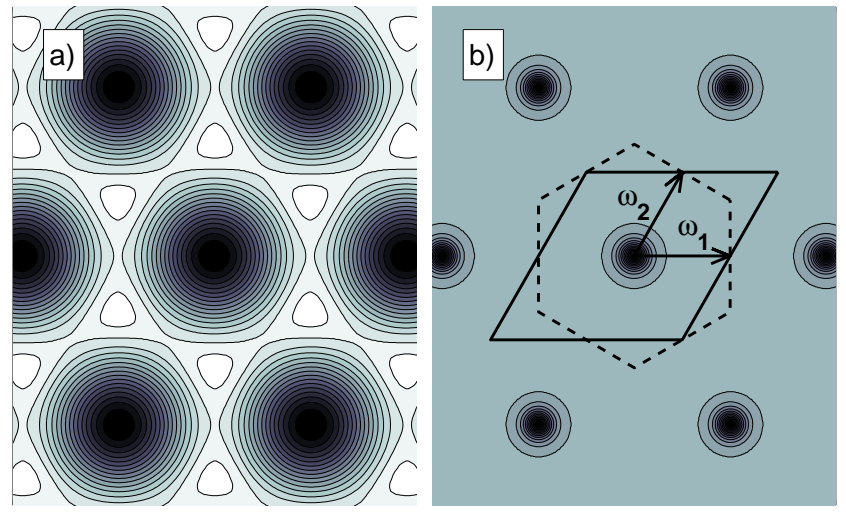

FIG. 1: Density distribution of the condensate in the $x-y$ plane computed by solving Eq. (2) for (a) $g=0$, (b) $g=300$. In (b) the dashed hexagon is the Wigner-Seitz cell, while the solid parallelogram is the computation box (see text). Darker regions correspond to lower density.

the axial direction from the radial one, therefore reducing to a 2-dimensional problem with an effective coupling constant $g_{2 D}$ [14]. In addition, our discussion can be conveniently reformulated in terms of dimensionless quantities by using harmonic oscillator units, i.e., $\hbar \Omega, \Omega$, and $l_{\Omega}=\sqrt{\hbar / m \Omega}$ for energy, frequency, and length respectively. The length $l_{\Omega}$ also fixes the equilibrium intervortex distance, according to the Feynman expression for the vortex density $n_{v}=m \Omega / \pi \hbar=1 / \pi l_{\Omega}^{2}$ [15]. Having to deal with an infinite periodic vortex lattice, we fix the average particle density $\langle n\rangle$ in terms of the number $N_{\text {cell }}$ of atoms per lattice cell, $\langle n\rangle=N_{\text {cell }} / A_{\text {cell }}$, where $A_{\text {cell }}=1 / n_{v}=\pi$ is the cell area. The corresponding dimensionless stationary Gross-Pitaevskii equation in the rotating frame is hence

$$
\mu \psi=\left[\frac{\left(-i \boldsymbol{\nabla}-\boldsymbol{e}_{z} \wedge \boldsymbol{r}\right)^{2}}{2}+g|\psi|^{2}\right] \psi,
$$

where $\mu$ is the chemical potential, $\boldsymbol{e}_{z}$ is the unit vec- 
tor along $z$, and the dimensionless coupling constant $g=\pi g_{2 D}\langle n\rangle / \hbar \Omega=g_{2 D} N_{\text {cell }} m / \hbar^{2}$ is the only parameter governing the equation. The normalization is $\int|\psi|^{2}=1$, where $\int \equiv \int_{A_{\text {cell }}} \mathrm{d} \boldsymbol{r}$ means integration over a single cell.

Due to the presence of the rotational term $\boldsymbol{e}_{z} \wedge \boldsymbol{r}$, Eq. (11) is not spatially periodic. However, in the spirit of the magnetic field analogy discussed in Refs. 7, 10], one can perform a gauge transformation which turns the symmetric effective vector potential $\boldsymbol{A}=\boldsymbol{e}_{z} \wedge \boldsymbol{r}$ into a periodic function $\boldsymbol{A}^{\prime}=\boldsymbol{A}-\boldsymbol{\nabla} \Lambda$. We choose $\Lambda=S_{T}$, where $S_{T}$ is the (non-periodic) phase associated with the Tkachenko (non-periodic) velocity field $\boldsymbol{v}_{T}=\boldsymbol{\nabla} S_{T}$ used by Tkachenko to describe the vortex lattice of an incompressible fluid [9]. With such a choice, indeed, one finds $\boldsymbol{A}^{\prime}=\boldsymbol{e}_{z} \wedge \boldsymbol{r}-\boldsymbol{v}_{T}=-\boldsymbol{v}_{T}^{\prime}$, where the Tkachenko velocity $\boldsymbol{v}_{T}^{\prime}$ in the rotating frame is now periodic [9]. We therefore write $\psi=\tilde{\psi} e^{i S_{T}}$ and substitute this expression into Eq. (11), finally obtaining

$$
\mu \tilde{\psi}=\left[\frac{\left(-i \boldsymbol{\nabla}+\boldsymbol{v}_{T}^{\prime}\right)^{2}}{2}+g|\tilde{\psi}|^{2}\right] \tilde{\psi},
$$

which admits periodic solutions. The explicit expression of $\boldsymbol{v}_{T}$ is conveniently written using the complex variable representation $z=x+i y, v=v_{x}+i v_{y}$ [16]. The lattice geometry is determined by the half-periods $\omega_{1}$ and $\omega_{2}$ (see Fig. 1(b)) which, due to the constraint $A_{\text {cell }}=\pi$, are related by $\operatorname{Im}\left(\omega_{1}^{*} \omega_{2}\right)=\pi / 4$. One then has $v_{T}\left(z^{*}\right)=i[\zeta(z)+\alpha z]^{*}$, where $\zeta(z) \equiv \zeta\left(z ; \omega_{1}, \omega_{2}\right)=$ $1 / z+\sum_{j k}^{\prime}\left[1 /\left(z-z_{j k}\right)+1 / z_{j k}+z / z_{j k}^{2}\right]$ is the quasi-periodic Weierstrass $\zeta$-function [17]. Here the primed summation excludes the term $j=k=0, z_{j k}=2 j \omega_{1}+2 k \omega_{2}$ are the vortex positions, and $\alpha=\left[\omega_{1}^{*}-\zeta\left(\omega_{1}\right)\right] / \omega_{1}$ is chosen to guarantee the periodicity of $v_{T}^{\prime}\left(z, z^{*}\right)=v_{T}\left(z^{*}\right)-i z$. Correspondingly, the Tkachenko phase is given by $S_{T}\left(z, z^{*}\right)=$ $\arg \sigma(z)+\operatorname{Im}\left(\alpha z^{2} / 2\right)$, where the Weierstrass $\sigma$-function obeys $\partial_{z} \sigma(z)=\zeta(z) \sigma(z)$.

In the following, we will determine the numerical solution of the GP equation in the periodic Tkachenko gauge, Eq. (2), for arbitrary values of $g$. Since the ground state solution of Eq. (2) can acquire a non-vanishing phase, the velocity field associated with $\psi$ does not coincide in general with $\boldsymbol{v}_{T}$, although the deviations are always found to be very small. Actually, the velocity field coincides with $\boldsymbol{v}_{T}$ not only in the incompressible regime $(g \rightarrow \infty)$ but also in the LLL limit $(g \rightarrow 0)$. Indeed the LLL wave function can be written in the form $\underline{6}$ ]

$$
\psi_{\mathrm{LLL}}=\prod_{j k}\left(z-z_{j k}\right) e^{-|z|^{2} / 2}=\sigma(z) e^{\alpha z^{2} / 2} e^{-|z|^{2} / 2}
$$

and hence, by using the explicit expression for $S_{T}$ reported above, one remarkably gets $\psi_{\mathrm{LLL}}=\left|\psi_{\mathrm{LLL}}\right| e^{i S_{T}}$.

We have solved Eq. (2) by restricting the calculation to the parallelogram given by the lattice vectors (see Fig. 1(b)) and imposing periodic boundary conditions. After discretizing the continuous problem on a triangular mesh, we used the finite element method [18] to find

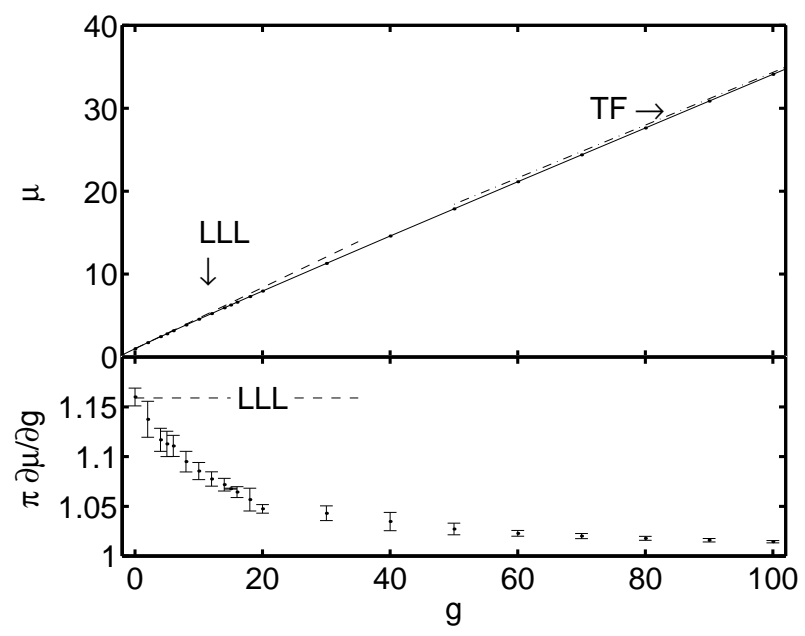

FIG. 2: Upper panel: numerically computed chemical potential $\mu$ (solid line) as a function of $g$. The dashed and dash-dotted lines are the LLL and TF predictions respectively. Lower panel: derivative $\pi \partial \mu / \partial g$. Numerical results (points with error bars) reproduce the correct behaviour in both the LLL and TF regimes.

solutions by means of imaginary time propagation. The energies of different lattices are calculated by varying the lattice vectors at constant cell area. We verified that the triangular geometry (where $\alpha=0$ ) is the favourite one for any value of $g>0$, while for $g=0$ any lattice with $A_{\text {cell }}=\pi$ has the same energy, reflecting the infinite LLL degeneracy. For $g<0$ we find that the system is unstable, as evident from its negative compressibility. In Fig. [ the equilibrium density profile extracted from the numerical solution of Eq. (2) for $g \rightarrow 0^{+}$and $g=300$ is presented. Vortices are characterized by holes in the density, whose size increases by decreasing the interaction. When $g \gg 1$, the density is uniform everywhere except in a very narrow region of size much smaller than the inter-vortex distance and the TF approximation applies. On the contrary, if $g \ll 1$ the vortex core radius becomes comparable to $l_{\Omega}$ and the density profile is highly structured (density maxima and saddle points alternate on the boundary of the hexagonal Wigner-Seitz cell).

It is also interesting to study the behaviour of the chemical potential $\mu$ as a function of $g$. Making a perturbative expansion around the LLL triangular lattice, one can prove that $\mu$ is linear in $g$ for $g \rightarrow 0$, i.e., $\mu \sim 1+\beta g / \pi$ [19] with $\beta=\int\left|\psi_{\mathrm{LLL}}\right|^{4} /\left(\int\left|\psi_{\mathrm{LLL}}\right|^{2}\right)^{2}=1.1596$ [12]. By using our numerical solution, the equation of state $\mu=\mu(g)$ can be calculated for any value of $g$, providing the bridge between the $g=0$ result and the TF relation $\mu=g / \pi$, valid for $g \gg 1$. Since these two opposite limits both exhibit a linear dependence with a different slope, the transition region shows a non linear behaviour. Deviations from linearity are however very small. The results are reported in Fig. 2] where we also plotted the derivative $\partial \mu / \partial g$ to highlight the transition. 


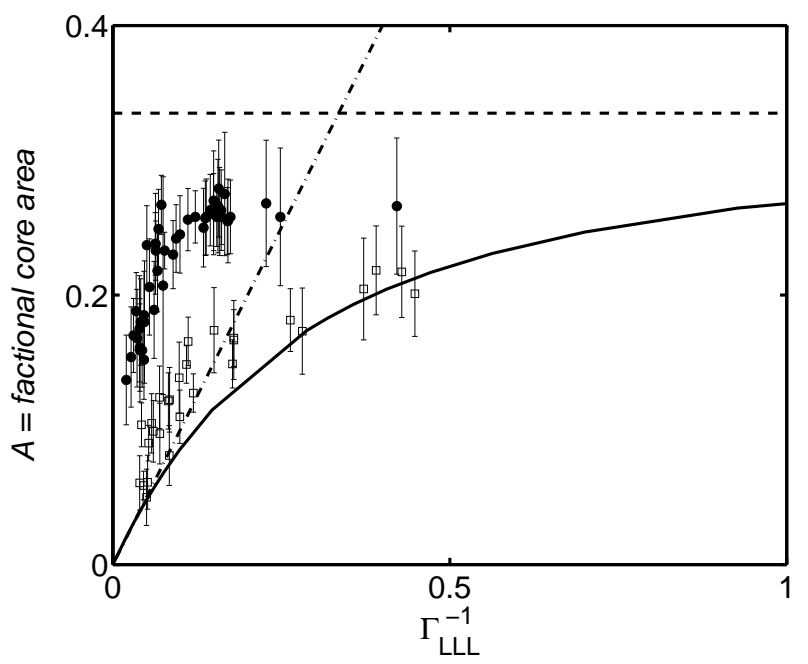

FIG. 3: Fractional vortex core area $\mathcal{A}$ as function of $\Gamma_{L L L}^{-1}$. Solid line: numerical solution of Eq. (2). Dashed line: LLL $\left(\Gamma_{L L L}^{-1} \rightarrow \infty\right)$ limit from Eq. (2). Dash-dotted line: TF $\left(\Gamma_{L L L}^{-1} \rightarrow 0\right)$ limit $\mathcal{A} \simeq 4 \xi^{2}$. Points with error bars: experimental results from Ref. [22] (see text).

The behaviour of $\mu(g)(g \propto\langle n\rangle)$ can be used to evaluate the coarse grained density profile $\langle n\rangle$ of the trapped rotating gas in terms of the residual effective potential $V_{\text {eff }}$, through the local density relationship $\mu(\langle n\rangle)+V_{\text {eff }}=$ const. In particular, for the experimentally relevant case of harmonic trapping, one notices that the quasi-linear behaviour of $\mu(g)$ implies the density profile to be an inverted parabola [20].

Another important quantity to study is the vortex core size. In the TF limit this length scale is fixed by the healing length $\xi=1 / \sqrt{2 \mu}$, while in the LLL limit, where $\xi$ diverges, the core size saturates to a fraction of the intervortex distance [5, 21]. Several definitions of the vortex core radius $r_{v}$ are possible. For a detailed comparison with experiments, we follow the procedure of Ref. [22], where $r_{v}$ is defined as the mean square root radius of the Gaussian which better fits the function $n_{\max }-n(\boldsymbol{r})$, evaluated on a single lattice cell 23]. Our numerical results are reported in Fig. 3] where we plot the fractional core area $\mathcal{A}=\pi r_{v}^{2} / A_{\text {cell }}=r_{v}^{2}$ as a function of the inverse of the LLL parameter $\Gamma_{\text {LLL }}=(\mu-1) / 2$ 22]. For $\Gamma_{\text {LLL }} \gg 1$ the vortex core radius follows the TF behaviour $\left(r_{v} \simeq 1.98 \xi\right)$, while for $\Gamma_{\text {LLL }} \rightarrow 0$ it reaches the limiting value $\mathcal{A}=0.34$. The latter result is not far from the value $\mathcal{A}=0.30$ derivable using the Gaussian fit definition with the circular cell approximation of Ref. [5].

Concerning the comparison with experimental results 22], two data sets are presently available. They differ in the procedure employed for the expansion, which is required to reach a sufficient resolution in the imaging process. The first set (open squares in Fig. 3) gives the fractional core area as measured during an expansion which preserves the vertical size, while the second one (filled cir-

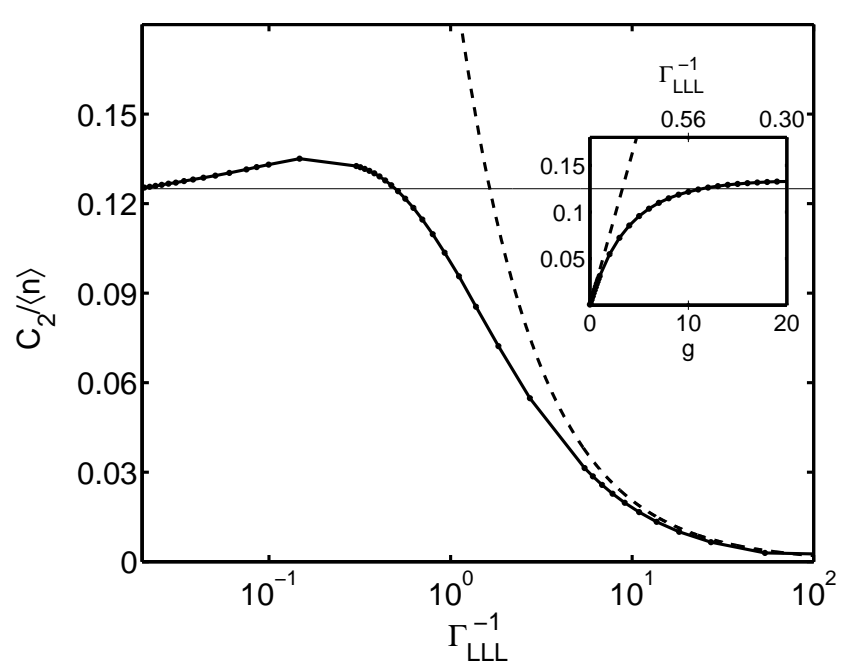

FIG. 4: Shear modulus $C_{2} /\langle n\rangle$ as a function of $\Gamma_{L L L}^{-1}$. Points connected by the solid line: numerical solution of Eq. (2). Dashed line: LLL prediction of Ref. [11]. Thin solid line: TF prediction of Ref. 25]. In the inset the same data are plotted as a function of $g$ (bottom label) and $\Gamma_{L L L}^{-1}$ (upper label).

cles) is obtained allowing also for some axial expansion. Being similar to a 2D scaling, the first procedure is expected to preserve the initial value of the fractional area $\mathcal{A}$ 24] and indeed the corresponding experimental data are in good agreement with theory. The second technique, involving an additional axial expansion, brings instead $\mathcal{A}$ closer to the LLL limiting value, since it lowers the value of the effective $2 \mathrm{D}$ coupling constant.

We finally studied the elastic shear modulus $C_{2}$. This quantity, related to the energy variation due to a shear distortion of the lattice [25], is a crucial ingredient for the determination of the frequencies of Tkachenko modes 11, 26, 27, 28, 29]. In order to extract this coefficient, we calculate the energy for different lattice shapes, starting from the triangular lattice and then varying one of the lattice vectors at fixed cell area: $\omega_{2}^{\prime} \rightarrow \omega_{2}+\epsilon \omega_{1}$. The coefficient $C_{2}$ is then given by $C_{2}=3 \delta \mathcal{E} / 4 \epsilon^{2}$, where $\delta \mathcal{E}$ is the (second order) variation of the energy per lattice cell.

Our numerical results are reported in Fig. 4 as a function of $\Gamma_{\mathrm{LLL}}$. In the TF limit $\Gamma_{\mathrm{LLL}}, g \gg 1$ we recover the result $C_{2} /\langle n\rangle=1 / 8$ [25], while in the LLL limit we find perfect agreement with the prediction of Refs. 11, 27], $C_{2} /\langle n\rangle \sim 0.1191 \mathrm{~g}$, which is a factor 10 larger than the value previously estimated in Ref. 28]. The figure shows that the transition between the two regimes is characterized by a maximum at $\Gamma_{\mathrm{LLL}} \simeq 5$. A consequence of this non monotonic behaviour is that the LLL regime of $C_{2}$ is reached at smaller values of $\Gamma_{\text {LLL }}$ with respect to the case of the vortex core (see Fig. 3). The results for the elastic shear modulus are relevant for the interpretation of the experiments of Ref. [30] on the Tkachenko oscilla- 
tions in harmonically trapped condensates. Indeed, one can average the predicted bulk values for $C_{2}$ over the TF profile of the trapped condensate, using a local density approximation (see for example Ref. 29]). This procedure allows to estimate the Tkachenko frequencies also in the intermediate regime between the TF and the LLL limit. In practice, for the highest rotation rate realized in the experiments, $\Omega / \omega_{\perp}=0.99$, where the gas is in a $2 \mathrm{D}$ regime and $\omega_{\perp}$ is the radial trapping frequency, we find that $C_{2}$ is lowered by only about $30 \%$ with respect to the $\mathrm{TF}$ value $\langle n\rangle / 8$. This means that the experimental data are still far from the LLL limit of the Tkachenko frequency, where the shear modulus would depend linearly on $g$ [1]. Furthermore, the predicted reduction of $C_{2}$ is not sufficient to explain the sizable discrepancy between the experimental measurements and the TF prediction [29]. Possible explanations of the remaining discrepancies are: (i) inadequacy of the local density approximation in the calculation of the Tkachenko frequencies, (ii) effects associated with the strong observed damping, (iii) deviations from linearity in the experimental excitation of vortex modes, and (iv) occurrence of anharmonic effects in the trapping potential.

In conclusion, we have investigated the bulk properties of a rotating condensate in the presence of exact compensation between the centrifugal potential and the harmonic trapping. To this purpose, the mean field GP equation has been solved by introducing a gauge transformation which explicitly exploits the periodic structure of the density distribution and of the velocity field in the rotating frame. We have systematically investigated the role of interactions exploring the transition between the Thomas-Fermi and the LLL regime. We have found that, while the size of the vortex core exhibits a smooth monotonic behaviour as a function of the interaction parameter, the elastic shear modulus is characterized by an intermediate maximum. The comparison with experiments reveals a good agreement as concerns the size of the vortex core and the static properties of the lattice, whereas we proved that the remaining discrepancies regarding the Tkachenko oscillations measured at the highest angular velocities cannot be attributed to LLL effects alone.

We are indebted with L.P. Pitaevskii, A.L. Fetter, J. Dalibard, and N.H. Lindner for fruitful discussions. We would like to thank E. Cornell and V. Schweikhard for valuable comments and sharing their data. C.T. would like to warmly thank M. Paolini for introducing him to the finite element method.

[1] K.W. Madison et al., Phys. Rev. Lett. 84, 806 (2000); J.R. Abo-Shaeer et al., Science 292, 476 (2001); P.C. Haljan et al., Phys. Rev. Lett. 87, 210403 (2001).

[2] P. Engels et al., Phys. Rev. Lett. 89, 100403 (2002).

[3] I. Coddington et al., Phys. Rev. Lett. 91, 100402 (2003).
[4] M. Cozzini and S. Stringari, Phys. Rev. A 67, 041602(R) (2003).

[5] G. Baym and C.J. Pethick, Phys. Rev. A 69, 043619 (2004).

[6] E.J. Mueller and Tin-Lun Ho, Phys. Rev. Lett. 88, 180403 (2002).

[7] N.K. Wilkin and J.M.F. Gunn, Phys. Rev. Lett. 84, 6 (2000); N.R. Cooper, N.K. Wilkin, and J.M.F. Gunn, Phys. Rev. Lett. 87, 120405 (2001).

[8] A.L. Fetter, J.A. Sauls, and D.L. Stein, Phys. Rev. B 28, 5061 (1983).

[9] V.K. Tkachenko, Zh. Eksp. Teor. Fiz. 49, 1875 (1965) [Sov. Phys. JETP 22, 1282 (1966)].

[10] Tin-Lun Ho, Phys. Rev. Lett. 87, 060403 (2001).

[11] E.B. Sonin, Phys. Rev. A 72, 021606(R) (2005).

[12] A. Aftalion, X. Blanc, and J. Dalibard, Phys. Rev. A 71, 023611 (2005).

[13] G. Watanabe, G. Baym, and C.J. Pethick, Phys. Rev. Lett. 93, 190401 (2004).

[14] By considering a system uniform along the $z$-axis with a vertical length $Z$, the effective $2 \mathrm{D}$ coupling constant is given by $g_{2 D}=g_{3 D} / Z$, where $g_{3 D}$ is the $3 \mathrm{D}$ coupling constant. If instead the motion along $z$ is squeezed into the lowest oscillator state, one has $g_{2 D}=g_{3 D} / a_{z} \sqrt{2 \pi}$, where $a_{z}$ is the axial oscillator length.

[15] R.P. Feynman, edited by C.J. Gorter, Progress in Low Temperature Physics (North-Holland, Amsterdam, 1955), Ch. 2.

[16] Such description proves to be extremely useful whenever $\boldsymbol{\nabla} \cdot \boldsymbol{v}=\boldsymbol{\nabla} \wedge \boldsymbol{v}=0$. Indeed, this implies the condition $\partial_{z} v=0$, i.e., $v$ turns out to be an analytic function of $z^{*}$. For example, for the particularly simple case of a single vortex, one has $v=i / z^{*}$.

[17] One has $\zeta\left(z+2 j \omega_{1}+2 k \omega_{2}\right)=\zeta(z)+2 j \zeta\left(\omega_{1}\right)+2 k \zeta\left(\omega_{2}\right)$ and $\zeta\left(\omega_{1}\right) \omega_{2}-\zeta\left(\omega_{2}\right) \omega_{1}=i \pi / 2$. See, for example, K. Chandrasekharan, Elliptic Functions (Springer Verlag, New York, 1985).

[18] C. Johnson, Numerical solution of partial differential equations by the finite element method, (Cambridge University Press, Cambridge, 1990).

[19] For $g=0$ one has $\mu=1$, corresponding to the zero point energy of the 2-dimensional harmonic trapping.

[20] The parabolic shape of the coarse grained density profile has been recently proven in the LLL regime starting from the exact solution of the GP equation [12, 13].

[21] U. R. Fischer and G. Baym, Phys. Rev. Lett. 90, 140402 (2003).

[22] I. Coddington et al., Phys. Rev. A 70, 063607 (2004).

[23] In Ref. 5] $r_{v}$ is instead defined as the mean square root radius of $n_{\max }-n$ on a single cell. However, such definition is not suitable for the TF regime, where it fails in reproducing the proportionality between $r_{v}$ and $\xi$.

[24] Castin and Dum, Eur. Phys. J. D 7, 399 (1999).

[25] G. Baym and E. Chandler, J. Low Temp. Phys. 50, 57 (1983).

[26] V.K. Tkachenko, Zh. Eksp. Teor. Fiz. 50, 1573 (1966) [Sov. Phys. JETP 23, 1049 (1966)].

[27] J. Sinova, C.B. Hanna, and A.H. MacDonald, Phys. Rev. Lett. 89, 030403 (2002).

[28] G. Baym, Phys. Rev. Lett. 91, 110402 (2003).

[29] M. Cozzini, L.P. Pitaevskii, and S. Stringari, Phys. Rev. Lett. 92, 220401 (2004).

[30] V. Schweikhard et al., Phys. Rev. Lett. 92, 040404 (2004). 